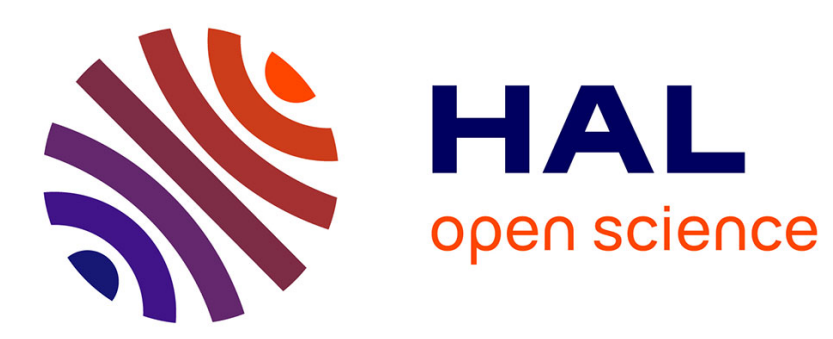

\title{
Fragments de la vie d'avant: une forme de récit dans des séances de soutien psychosocial pour des femmes réfugiées
}

Véronique Traverso

\section{To cite this version:}

Véronique Traverso. Fragments de la vie d'avant: une forme de récit dans des séances de soutien psychosocial pour des femmes réfugiées. Langage et Société, 2021, pp.83-113. halshs-03423950

\section{HAL Id: halshs-03423950 \\ https://shs.hal.science/halshs-03423950}

Submitted on 19 Nov 2021

HAL is a multi-disciplinary open access archive for the deposit and dissemination of scientific research documents, whether they are published or not. The documents may come from teaching and research institutions in France or abroad, or from public or private research centers.
L'archive ouverte pluridisciplinaire HAL, est destinée au dépôt et à la diffusion de documents scientifiques de niveau recherche, publiés ou non, émanant des établissements d'enseignement et de recherche français ou étrangers, des laboratoires publics ou privés. 
Pré-print

Fragments de la vie d'avant : une forme de récits dans des séances de soutien psychosocial pour des femmes réfugiées

Véronique Traverso, Institut Français du Proche-Orient / ICAR

L'interaction au carrefour du langage, du corps et de la société. Hommage à Charles Goodwin Langage \& Société 173, 2021/2, pages 83-113

Pour démarrer cet article sur le récit en hommage à Goodwin, je mettrai en parallèle deux citations dans lesquelles il en donne des éléments de définition. La première est issue de son article de 1984 "Note on story structure and the organization of participation". Elle conclut une première analyse du récit "le papier peint", produit au cours d'une conversation entre quatre amis, où la narratrice raconte, en présence de son mari, une gaffe qu'il a faite, alors qu'ils visitaient pour la première fois le nouvel appartement de leur amie Karen :

A multi-utterance turn [...], the body of the story, is preceded by specific moves by both the teller and the recipient: a preface offering to tell the story [...] and a request to hear the offered story [...]. At the conclusion of the story proper the participants engage in further talk relevant to it [...]. The body of the story contains a number of distinct sections. It begins with relevant background information [...] and then with moves to what will turn out to be the climax of the story. At this point, however, rather than producing what Don in fact said the speaker begins to provide further background information [...]. The story thus contains not only preface, background, and climax sections but also what may be called a parenthesis, a section of background information embedded (disjunctively) within the climax. (1984: 226)

Dans cette citation, le récit est décrit en termes de structuration globale, comme possédant une préface et un "corps" au sein duquel se succèdent des parties bien distinctes, informations d'arrière-plan, parenthèse, point culminant (" climax ").

La deuxième citation provient de l'article "Narrative as talk in interaction" publié dans le Handbook of narrative analysis (Georgakopoulou \& De Fina, 2015). Elle apparaît en conclusion d'une analyse dans laquelle Goodwin montre comment les participants manifestent, à travers l'organisation de leurs conduites verbales et corporelles, leur compréhension qu'un récit est en train d'émerger:

« [a narrative is] a field of action built collaboratively by structurally different actors using a variety of semiotic resources within face-to-face interaction (2015: 204)

Dans l'espace de réflexion et de découverte entre ces deux définitions, s'inscrit une grande partie de l'oeuvre de Goodwin. S'y expriment au moins trois perspectives: une analyse narrative somme toute assez classique - distinguant des composantes du récit (informations d'arrière-plan, parenthèse, "climax ") ; une description interactionnelle du récit comme un tour multi-unités, initié par une préface qui permet d'en négocier l'introduction et suivi par une reprise de l'alternance des tours de parole ; et une conception praxéologique et holistique du récit, comme une activité mettant en jeu la scène interactionnelle entière (et non seulement le narrateur ou la narratrice), les corps des participants (et non leur seule parole), leurs savoirs partagés, transmis, repris et transformés, ainsi que, plus largement, les positions et la construction d'identités que le récit occasionne et qui organisent la scène sociale. 
Dans la première partie de cet article, je présente ce qui constitue à mes yeux les apports majeurs de Goodwin aux approches interactionnelles du récit (multimodalité, participation et organisation sociale). Sur cette base, j'étudie ensuite une forme de récits récurrente au cours de séances de soutien psychosocial pour des femmes réfugiées syriennes dans une association à Beyrouth, des "récits d'avant". Dans ces récits, les narratrices évoquent des aspects de leur vie en Syrie, le plus souvent en comparaison avec leur vie actuelle au Liban. Je m'attache à caractériser ces récits et à les examiner à la lumière de la façon dont ils transforment à différents niveaux la situation dans lesquelles ils sont produits. La question qui m'intéresse in fine est ce qu'il résulte de ces transformations, dans l'interaction aussi bien que dans le cadre plus large où elles ont lieu. A travers une analyse multimodale inspirée de Goodwin, je cherche ainsi à voir ce que font les femmes en produisant ces récits d'avant.

\section{Le récit : un "champ d'action"}

Comme l'indiquent les citations ci-dessus, Goodwin développe une vision résolument interactionnelle du récit ${ }^{1}$ (Sacks, 1974, 1978, 1992 ; Jefferson, 1978, 1988 notamment). Cette conception examine le récit comme une activité collective, que les participants mettent en route puis arrêtent dans le fil de leurs échanges. La dimension collective d'un récit est particulièrement visible dans son introduction ainsi que dans les formes de contributions des interlocuteurs ${ }^{2}$. Introduire un récit dans l'interaction nécessite en effet d'obtenir des interlocuteurs la possibilité de produire un tour long (tour composé de plusieurs unités de tours), qui vient momentanément suspendre l'alternance des tours de parole, d'où l'usage de "préfaces ${ }^{3}$. La production du récit dans l'interaction est soutenue par les récepteurs à travers leurs accusés de réception et continueurs, mais aussi par leurs évaluations qui leur permettent d'exprimer, outre leur alignement sur l'activité, leur affiliation (Bonu 2001, Stivers 2008, Mandelbaum 2013) ${ }^{4}$. Une autre forme de contribution des récepteurs à la production du récit est la production d'une "second story", à travers laquelle le récepteur montre aussi sa compréhension de ce que le narrateur a raconté. Sacks décrit en ces termes les tâches du récepteur d'un récit :

Recipient of a story has as one business to display his understanding of it [...], and and/or to affiliate to it by showing its particular relevance to him. "Oh God love 'im" is one form such affiliation takes. Another form involves recipient telling a second story, in which recipient figures as teller had figured. (1978 : 261)

\footnotetext{
${ }^{1}$ Cette approche a fondamentalement revisité l'approche du récit des études narratologiques, basée sur la recherche de ses traits caractéristiques (enchaînement de propositions narratives, emploi des temps, marqueurs spatio-temporels, mise en intrigue) et ses questions telles que, par exemple, celle du "récit minimal". Voir des discussions sur ces caractéristiques structurales du récit, ainsi que ses caractéristiques interactionnelles (preface, tellability, evaluation) dans Filliettaz et Revaz (2006), et Traverso (à paraître). Mandelbaum (2023) propose une synthèse des descriptions interactionnelles du récit.

2 Sur ce point précisément, les descriptions conversationnelles du récit s'éloignent des propositions de Labov et Waletski (1967), qui ont été forgées sur la base de récits produits par des interviewés à qui l'intervieweur demande de raconter une expérience où sa vie a été mise en danger. Dans un tel format, contrairement à ce qui est le cas dans les conversations par exemple, les narrateurs n'ont pas à négocier l'introduction d'un récit, puisqu'ils sont d'emblée, comme le dit Goodwin, dans la position " of having the rights to an extended floor " (2015 : 198). Sur l'approche labovienne, voir notamment Bres 2001.

${ }^{3}$ Ainsi définies par Sacks : " It is an utterance that asks for the right to produce extended talk, and says that the talk will be interesting, as well as doing other things » (1992, t2 : 226), voir aussi Jefferson 1978.

4 Voir Goodwin 1986, Goodwin \& Goodwin 1987 sur "receipt tokens" et "assessments" et sur la valeur clôturante des évaluations.
} 
Ces caractéristiques succinctement rappelées ici forment le socle de l'approche du récit à partir de laquelle Goodwin propose des changements de perspective, apports et enrichissements. II ne semble pas que la recherche de caractérisations en propre du récit, comme distinct d'autres activités interactionnelles, ait été le moteur de ses propositions. Le récit apparaît plutôt dans son oeuvre comme un type d'activité parmi d'autres dont l'étude détaillée a permis l'approfondissement de la réflexion sur la nature et les formes de réception (recipientship), sur la participation et la variété de ses modes. Ainsi, ce qui frappe dans les études de Goodwin sur les récits est la façon dont il enrichit la vision de la scène interlocutive, dont il "affine la focale" sur des phénomènes invisibles jusque-là, ou en tout cas non décrits dans leurs détails, plus que la proposition d'une conception inédite qui s'inscrirait dans la longue tradition des études du récit. D'ailleurs, les récits qu'il étudie, dont nous avons quelques exemples dans la suite de cette section $(1.1,1.2)$, sont, à bien des égards, des récits canoniques, dans le sens où ils présentent les caractéristiques classiquement retenues: déplacement vers un autre temps, succession d'événements à partir d'une situation initiale rendue par une suite de propositions narratives, déroulement temporel sous-tendant une mise en intrigue, et chute ${ }^{5}$. Ils sont donc assez éloignés des récits moins classiques, moins unifiés et moins bien formés, très courants dans les conversations, regroupés aujourd'hui sous le nom de "small stories" (Georgakopoulou 2006, Bamberg \& Georgakopoulou 2008) : " an umbrella term that captures a gamut of undersrepresented narrative activities, such as tellings of ongoing events, future or hypothetical events, and shared (known) events, but it also captures allusions to (previous) tellings, deferrals of tellings, and refusals to tell » Bamberg \& Georgakopoulou $2008: 381)^{6}$. Le label est introduit pour se distinguer des "récits canoniques", que De Fina \& Tseng caractérisent en référence au modèle de labovien : "According to William Labov, [...] stories are recapitulations of past events, with a structure involving complications and resolutions, told by a narrator in order to make a point. These canonical narratives recount chronologically ordered events in the past, have well-defined beginnings/middles/ends, and usually revolve around significant incidents " (2003: 381). Les "petites histoires" incomplètes, mal formées ou fugaces, qui s'éloignent du récit "canonique", forment en fait une grande partie des récits ordinaires et quotidiens, et leur étude accompagne les recherches interactionnelles (par exemple Jefferson 1988, Traverso 1994, 1996).

Si les analyses de récits proposées par Goodwin ne reprennent pas les discussions sur ce qu'est ou n'est pas un récit, elles n'en constituent pas moins un apport majeur pour l'étude de cette activité interactionnelle, à travers des avancées relatives aux ressources sémiotiques, à la stratification des participants et à la construction d'organisations socio-interactionnelles. Ces aspects sont présentés séparément dans les trois sections qui suivent, ce qui, j'en ai bien conscience, entre en contradiction avec la vision que Goodwin s'est attaché à promouvoir, en

\footnotetext{
${ }^{5}$ Ces caractéristiques ont été beaucoup discutées et les recherches sur le récit dans les interactions sociales convergent pour considérer que nombre des récits qu'on y trouve ne remplissent qu'imparfaitement ces critères (voir parmi de nombreux autres Filliettaz \& Revaz, 2006, sur des récits produits dans des interviews avec des opératrices d'une usine pharmaceutique ; Traverso, 1994, 1996 sur les récits de la confidence ; Bercelli et al. , 2008 sur des récits en psychothérapie etc.).

${ }^{6}$ Dans son article de 2015, Georgakopoulou explicite le caractère militant de cette étiquette de "small story", (opposée à "big story", référant aux récits de vie, récits biographiques), dont l'introduction était liée à la nécessité de souligner l'existence d'un biais dans les recherches et d'opérer une contre-attaque sur les modèle dominants du récit (2015:256).
} 
révélant le constant maillage des différentes dimensions. Ce choix ne répond qu'à des exigences de clarté d'exposition.

\subsection{Les ressources sémiotiques}

Avec les notions de "situated activity systems" (1997) ou de "interactive fields" (2015: 203), Goodwin montre que sont mis en jeu dans l'interaction, non seulement les dimensions linguistiques, discursives et prosodiques, les corps et leur organisation les uns par rapport aux autres, les objets et les organisations spatiales, mais aussi des phénomènes interactionnels et des pratiques, au premier rang desquels se trouve l'organisation séquentielle ainsi que diverses formes d'actions spécifiques et reconnaissables (comme des réparations ou des expressions de désaccord par exemple). Un des exemples de ces systèmes où s'imbriquent les ressources sémiotiques est justement un récit, celui du papier peint, que Goodwin reprend de nombreuses fois au fil des années $(1984,2007,2015,2018)$ :

Extrait 1

[G:26:5:55] (Simplified Version)

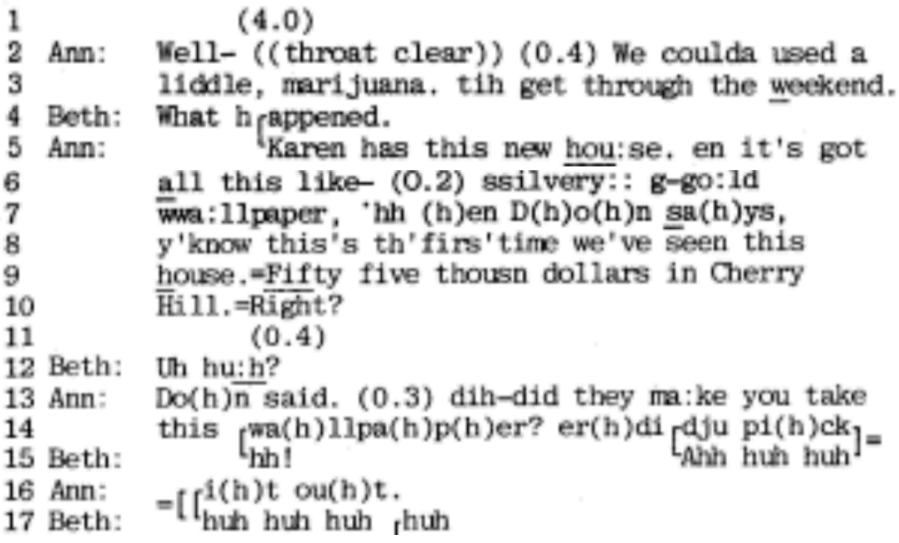

Repris de Goodwin 1984, pages 225-226

Goodwin détaille comment Don, le mari de la narratrice, qui est présent pendant qu'elle raconte la gaffe qu'il a faite, participe activement, bien que silencieusement, au récit, par la façon dont il organise visiblement son corps, la direction de son regard, les expressions de son visage et son rire. On le voit notamment dans le tour de la ligne 14, qui est le point culminant de I'histoire, où la narratrice insère des syllabes de rires à l'intérieur de syllabes de mots. Simultanément, Don produit, dans une autre modalité, des mimiques de rires, qui suivent, syllabe par syllabe, le rire vocal d'Ann. Goodwin décrit précisément comment l'expression du visage de Don s'amplifie au cours de "wa(h)Ilpa(h)p(h)er" (extrait 2), ce qui résulte en un rire produit simultanément par deux corps séparés (2018:132).

Extrait 2

did they ma:ke

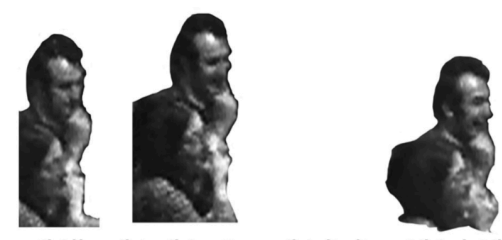

you take this wa(h)\|pa(h)p(h)er? =er(h)di dju pi(h)ck i(h)t ou(h)t. 
Ces analyses très détaillées des ressources utilisées par les participants conduisent à élargir considérablement la conception que l'on peut avoir des formes de contributions au récit, en prenant en compte non seulement la parole des locuteurs mais aussi leur corps. Elles invitent aussi tout naturellement à revisiter la question de la participation, et notamment les instances de locuteur(s) et récepteur(s) engagés dans l'activité narrative.

\subsection{Participants pluriels et stratification des instances}

Un autre très important aspect de l'apport de Goodwin à l'étude des récits est d'avoir discuté, dans le cadre de sa propre recherche, des propositions émanant d'autres traditions, et d'avoir combiné différentes dimensions, notamment la dimension séquentielle et la dimension énonciative. Sa réflexion sur ce point repose sur une élaboration des propositions de Goffman (1981) concernant la déconstruction des participants, ainsi que sur une relecture "corporalisée" de la polyphonie de Voloshinov (Marxisme et la philosophie du langage). Dans plusieurs articles (par exemple Goodwin 2006, 2015), il discute des limites de ces deux conceptions et propose sa propre vision, qui est une vision temporalisée (et non statique), d'une granularité extrêmement fine (et non globale et générique), multimodale (et non logocentrée), et qui intègre "dans le même monde"7 l'ensemble des participants à la scène interactionnelle.

Dans son article de 2007 concernant le récit du papier peint, il montre que, dans une analyse goffmanienne, la parole d'Ann engloberait une riche série d'instances (auteur, animateur, etc.) alors que les autres participants ne seraient que des entités figées dans un statut fixe et immuable (récepteur, participant officiel, adressé ou non, etc.). En contraste, sa propre analyse fait apparaître, d'une part, que Don, personnage mis en scène et récepteur du récit d'Ann, est aussi bel et bien un co-producteur du récit, et d'autre part, que les modes de participation des "récepteurs" à la production du récit, ainsi que les ressources qu'ils utilisent, se modifient au fil du déploiement temporel du récit.

La description de ces ressources et de la complexité des contributions des différents participants va encore plus loin dans l'analyse que Goodwin fait des récits de son père Chil, aphasique suite à un AVC, et ne disposant plus sur le plan linguistique que des trois mots "oui", "non", "et" et d'un certain nombre de syllabes dénuées de sens, produites avec des prosodies variées (Goodwin et al. 2002). Son analyse d'un récit de Chil à propos d'un tremblement de terre (Goodwin 2004, 2013) dans le cadre d'une réunion familiale met en évidence, non seulement comment Chil parvient à faire raconter une "'second story" (Sacks 1992), mais aussi comment, par les expressions de son visage et de son propre corps, il se fait co-narrateur du récit que produit verbalement Helen. $A$ un certain moment du récit (au moment où il est question d'un tableau qui manque de tomber sur un bébé à cause d'une secousse), Chil manifeste son désaccord avec Hélène :

Extrait 3

\footnotetext{
${ }^{7}$ Repris de Goodwin dans sa critique de Footing de Goffman : «In Footing, instead of collaborating together to build talk, speakers and hearers inhabit separate worlds » (2007: 28).
} 


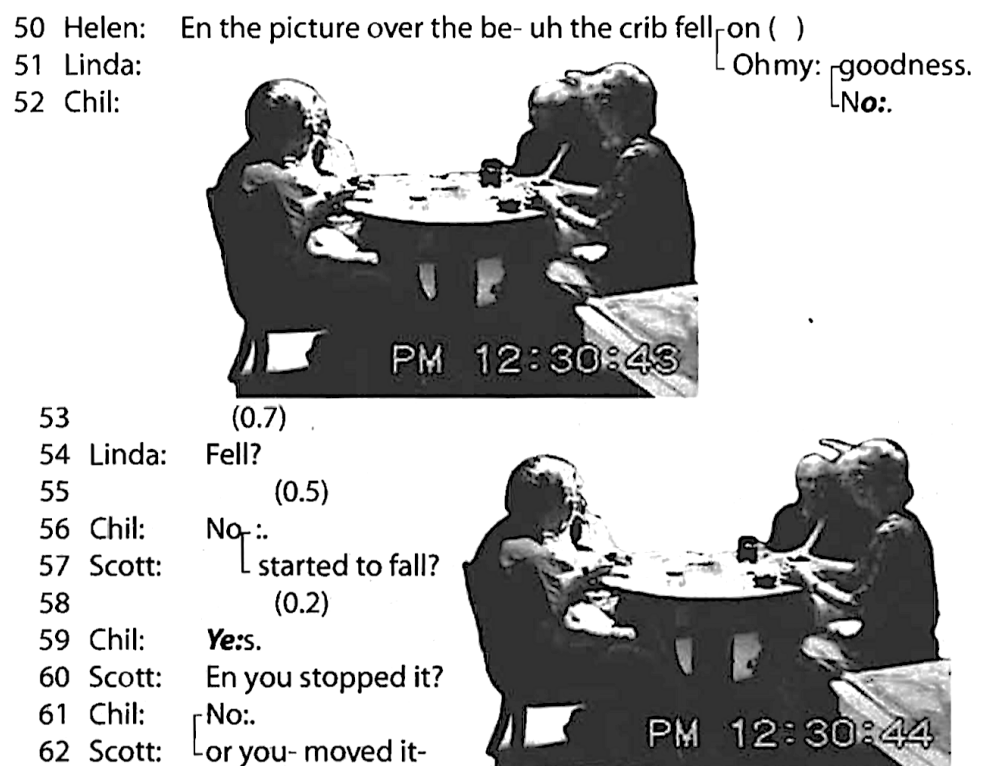

Repris de Goodwin 2004, page 164

Alors qu'Helen affirme que le tableau est tombé (I. 50), Chil exprime un désaccord ("no:", I. 52), tout en levant sa main au-dessus de sa tête, avant de regarder Helen puis Linda (qui vient de formuler une évaluation, I. 51). Le geste produit à cet emplacement séquentiel où une explication du désaccord serait normativement attendue, fonctionne comme référant à une version alternative de l'événement, ce qui conduit les autres participants à la rechercher ("started to fall?", I. 57), et qui aboutit finalement à une production collective de la version de I'histoire voulue par Chil. Avec ses analyses des récits de Chil, Goodwin s'éloigne encore davantage des références de départ (Goffman et Voloshinov), en mettant en évidence une très profonde imbrication des instances énonciatives et des formes de participation. II parle de "locuteur distribué" ("distributed speaker") pour chacun d'entre nous qui, comme Chil, sommes capables d'incorporer de façon co-opérative dans nos propres actions des ressources provenant des autres ( " co-operatively incorporate in our own actions resources provided by others ", $2018: 103)$.

\subsection{La construction d'organisations socio-interactionnelles à travers l'activité narrative}

Le dernier aspect qu'il importe de souligner à propos des études de Goodwin sur les récits concerne la façon dont ils reflètent et contribuent à la construction d'organisations sociales. Il élabore ses réflexions sur le sujet avec Marjorie Goodwin, à partir des travaux de cette dernière sur les disputes chez les jeunes Africains-Américains.

Ensemble, ils étudient par exemple comment un type spécifique d'histoires racontées construit une organisation sociale spécifique du groupe de filles, en mettant les participantes dans des places qui les poussent à agir de telle ou telle manière. A titre d'exemple, si Bea dit à Julia publiquement que Terry a émis des propos négatifs concernant Julia, cette histoire rapportée mettra Julia en position de "personne offensée", ce qui la conduira à vouloir accuser et demander des comptes à Terry. Ces "instigating stories" (M. Goodwin 1982) mettent en place des protagonistes et des événements évoqués d'une façon qui s'articule fortement avec des positions qu'elles poussent les filles du groupe à endosser et des actions sociales qu'elles les poussent à accomplir. En ce sens, elles construisent et transforment les organisations 
sociales et les identités des participants ${ }^{8}$. Cette notion de transformation revêt une importance majeure dans les derniers travaux de Goodwin. L'auteur voit ce processus à l'oeuvre à différents niveaux, par exemple lorsque les participants reprennent et transforment dans un tour successif des éléments et parties d'un tour précédent (les "substrates", Goodwin 2018), dans le fait qu'un récit transforme in situ (c'est-à-dire dans le temps de sa production) l'organisation socio-interactionnelle en place, aussi bien que dans la transformation en retour que l'interaction in situ va avoir sur les actions des participants, et sur eux-mêmes :

The process of participating in the interactive field created by a narrative - performing transformative operations on it that display understanding and build appropriate, consequential subsequent action - simultaneously transforms those who perform such operations. (2015: 216)

A nouveau, on se retrouve ici dans des approches focalisées, non sur le récit en tant que suite de propositions, ni sur la réalité, vérité ou justesse des faits racontés, mais sur ce qu'il fait dans la situation où il est produit, pour, avec et entre les personnes présentes.

\section{S'opposer, s'échapper, redéfinir le cadre en racontant dans un collectif}

Dans le cadre du projet sur les "Dimensions linguistiques et communicatives dans l'assistance aux réfugiés et personnes déplacées au Liban" ${ }^{\prime \prime}$, et en continuité avec une série d'études sur la construction des positionnements identitaires dans des réunions (Traverso 2003, 2014, 2017), l'étude qui suit entend décrire ce qui, dans le contexte très spécifique de séances de soutien psychosocial à destination de femmes précaires et réfugiées, apparaît comme un type particulier de récit. Au-delà de caractériser ces récits, je chercherai à montrer comment leur production transforme le cadre en place. Enfin, je cherche à comprendre vers quoi mènent ces transformations, non seulement dans l'ici et maintenant de l'interaction (à travers les formes d'enchaînements séquentiels qu'ils suscitent), mais surtout dans le contexte plus large de la séance où ils prennent place et de l'organisation sociale que les séances de soutien psychosocial reflètent.

\subsection{Les séances de Life skills}

Les Life skills and awareness sessions sont présentées par les organismes humanitaires comme " tout type de soutien local ou extérieur visant à protéger ou à favoriser le bien-être psychosocial et/ou à prévenir ou traiter les troubles mentaux " (InterAgency Standing Committee IASC, 2007). Dans I'ONG Nina, où les séances ont été enregistrées, les femmes viennent se former aux artisanats textiles. Leur inscription rend également obligatoire de suivre une session de Life skills, où leur présence est d'ailleurs contrôlée. Une psychologue ou une travailleuse sociale (salariée de l'association ou extérieure) anime ces séances.

Les récits que j'étudie sont donc produits dans un cadre qui, en lui-même, opère des attributions identitaires ${ }^{10}$ : le fait de consacrer une séance au droit des femmes, au stress ou à I'hygiène caractérise en effet les réceptrices comme 1) étant susceptibles de souffrir de

\footnotetext{
${ }^{8}$ Les identités et les positionnements sont ainsi abordés de façon fondamentalement interactionnelle, c'est-àdire conçues comme mises en place in situ, de façon à la fois séquentielle et relationnelle (voir entre autres Antaki \& Widdicombe 1998 ; Greco, Mondada et Renaud 2014).

${ }^{9}$ http://www.icar.cnrs.fr/sites/projet-remilas/remilas-liban/. Ce projet a été financé par I'IFPO (Beyrouth) et I'Université Saint Joseph (Beyrouth).

${ }^{10}$ Greco, Mondada, Renaud Traverso 2014, Traverso 2017, Marranconi \& Kerbage 2007, Kerbage \& Traverso 2020.
} 
problèmes liés à ces thématiques, 2) ne disposant pas des savoirs, ou du moins nécessitant des informations sur ces questions. Je me focalise dans cet article sur une séance consacrée à I'hygiène à laquelle assistent deux groupes de femmes qui se forment à deux techniques de broderie (broderie mécanique, aghabâni et broderie manuelle). Les deux formatrices de broderie rejoignent la séance en cours de route. Le groupe est installé autour d'une grande table (une des deux formatrice est hors champ). L'animatrice (psychologue) est installée en bout de table ; les femmes qui participent à la séance sont réparties selon leur ordre d'arrivée par groupe d'activité. La configuration qui en résulte est la suivante : de part et d'autre de la table se trouvent, à la droite de la psychologue, le groupe de aghabâni, et à sa gauche le groupe de broderie manuelle.

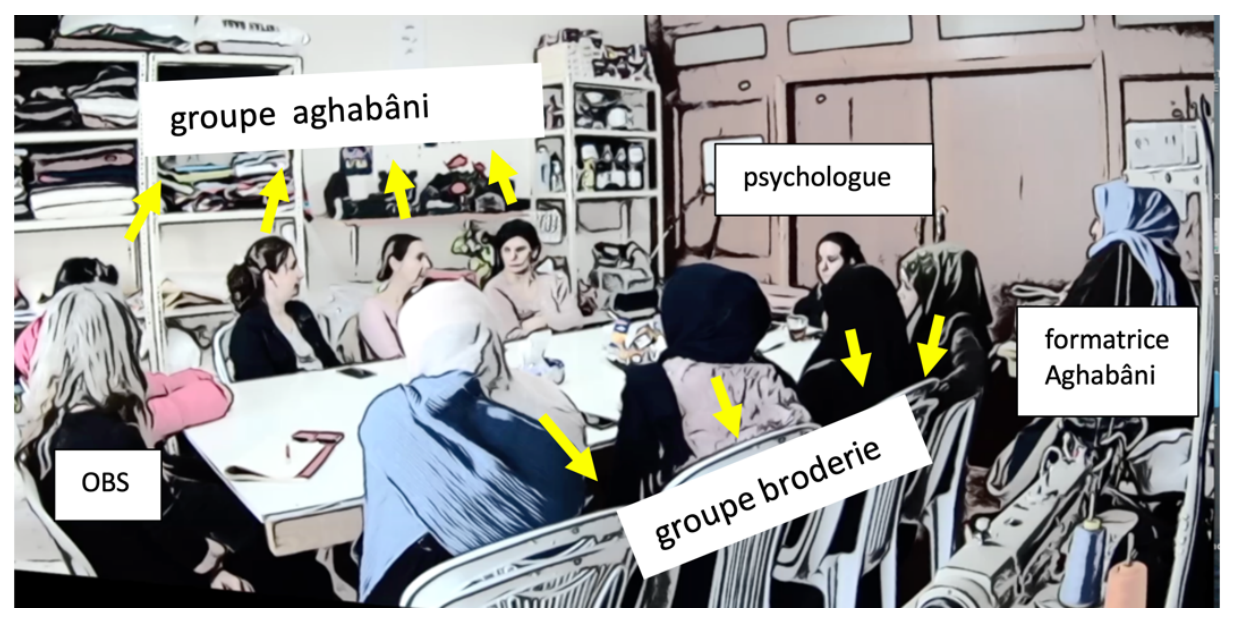

Image 1

Outre que par leur artisanat, les deux groupes se distinguent aussi par le fait la plupart des femmes du groupe de aghabâni sont des libanaises précaires, sauf une déplacée iraquienne alors que toutes les femmes du groupe de broderie manuelle sont des femmes syriennes.

\subsection{Les récits d'avant}

Les "récits d'avant" évoquent le pays quitté. Ce sont des récits d'avant la guerre (d'Iraq ou de Syrie), d'avant l'exil. Ils ne racontent pas des faits ou des événements spécifiques ayant eu lieu à un moment bien localisé du passé ; ils concernent des habitudes, des routines de la vie dans le pays quitté. En les racontant, les femmes soulignent les différences de modes et de conditions de vie entre la Syrie et le Liban et font valoir ce qui était mieux avant, là-bas.

Ces récits se distinguent des récits les plus fréquemment analysés dans le champ d'étude de la migration (voir entre autres Canagarajah 2003, Pestre 2010, Jacquemet 2010, d'HalluinMabillot 2012, Maitilasso 2014, Mekdjian 2016, Chambon 2018, Saglio-Yatzimirski 2018, Chambon \& Traverso à paraître). En effet, ils ne sont pas une des variantes et reprises du "récit du réfugié" tel qu'il doit être produit pour les organismes traitant des demandes d'asile ${ }^{11}$ ou tel qu'il est préparé dans les associations aidant les migrants pour leur dossier de demande. Ils ne sont pas non plus des récits sollicités dans des entretiens ou des focus groupes ${ }^{12}$, autre

\footnotetext{
${ }^{11}$ Tels que, pour la France, I'Office français de protection des réfugiés et apatrides ou la Cour National du Droit d'Asile.

${ }^{12}$ A propos desquels Maitilasso rapporte l'anecdote suivante : "Tu veux que je te raconte mon histoire ? Je peux le faire, je l'ai déjà fait beaucoup de fois", m'explique en souriant Thierno [...] quand je lui parle de ma recherche et de mon désir d'échanger avec lui à propos de son parcours migratoire. L'histoire de vie de Thierno se présente comme un véritable produit, l'expression d'une performance littéraire reproductible à chaque fois
} 
grande catégorie de récits traités dans le champ d'étude de la migration. En effet, les récits d'avant ne sont pas sollicités, ils surgissent ; ils viennent accomplir dans l'interaction quelque chose qui est le fait propre des narratrices, et non leur tentative de se conformer aux attentes (du chercheur) ou aux exigences (de l'officier de protection). Ils se tiennent dans un cadre où ils ne sont pas spécifiquement attendus ni demandés, mais où ils ont toutefois la possibilité d'être produits.

Sur le plan formel, ils sont bien loin de comporter toutes les caractéristiques du récit canonique rappelées ci-dessus. Une des marques linguistiques qui les caractérise consiste en des énoncés introductifs comportant des marques temporelles et spatiales opérant une rupture avec l'ici et maintenant de l'interaction ("quand j'étais/on était à...", "autrefois..."). Ils sont le plus souvent très brefs, et ne répondent pas du tout au critère de "mise en intrigue", c'est-à-dire à une dynamique de type logique (relation de causes à effets) sous-tendue par une organisation temporelle (propositions ordonnées). La dynamique qui les soutient réside dans la mise en contraste, la mise en opposition. Une dernière de leurs caractéristiques importantes est qu'ils prennent place dans des situaitons plurilocuteurs, et qu'ils sont euxmêmes collectifs, même si la plupart du temps une seule locutrice en produit la forme verbale. En ce sens, ils sont véritablement un champ d'action (Goodwin 2015). Dans la lignée des recherches de Goodwin rappelées ci-dessus, j'analyse deux de ces récits, qui présentent à la fois des similarités et des différences. Je me concentre sur la façon dont ils émergent, et dont ils conduisent à réorganiser le groupe de participantes.

\subsection{La mise en place d'un récit collectif : "on avait une grande maison"}

L'organisation de la séance de Life Skills consacrée à l'hygiène se fait en deux temps : après l'annonce de son thème, la psychologue organise un tour de table au cours duquel chaque femme à son tour donne son point de vue sur ce que cette notion recouvre et sur son importance (Traverso \& Kerbage 2020). Puis, elle récapitule et énonce des principes généraux d'hygiène, phase qui peut occasionner des discussions et des désaccords (Idem).

Maha, désignée pour parler la première dans le tour de table, aborde les difficultés qu'elle a à garder ses enfants propres. A la fin de sa prise de parole, au moment où elle s'apprête à passer le tour à sa voisine de droite (image 1 ci-dessous), une autre participante (assise en face d'elle), Maïssa, prend le tour. L'extrait reproduit commence à la fin de la prise de parole de Maha, au moment où Maïssa puis la psychologue, produisent quelques tours, entérinant la fin de cette première prise de parole et formulant leur accord (Maïssa, ligne 4) et proposant un énoncé généralisant (la psychologue, lignes $5,7,9)$ :

Extrait $4^{13}$

qu'on le désire ; un produit pour lequel il existe une forte demande de la part des journalistes, ethnographes et activistes des droits humains " (2014:1). Ma propre expérience dans l'association Nina reflète aussi cet état de fait : autant les participantes (formatrices et bénéficiaires) étaient déconcertées que je ne leur demande pas de me raconter leur vie mais d'enregistrer les séances de formation et de Life Skills, autant, lorsque j'ai enfin réalisé des entretiens (sur leur expérience de formatrice en artisanat textile), elles se sont retrouvées en terrrain connu, et j'ai compris à quel point ce format leur était familier, et d'ailleurs agréable.

13 Je remercie Sophie Harfouche et Maha Barakat pour la transcription des corpus. Toute ma reconnaissance va aussi à l'ensemble des personnes de l'association Nina, qui m'ont accueillie parmi elles pendant une année et ont accepté les enregistrements. 
Toutes les interactions se tiennent en arabe. Pour alléger la lecture, dans certains extraits, seule la traduction française apparaît. La langue originale, sous forme de transcription phonétique, est reproduite lorsque cela s'impose pour indiquer plus finement la séquentialité du tour et la multimodalité.
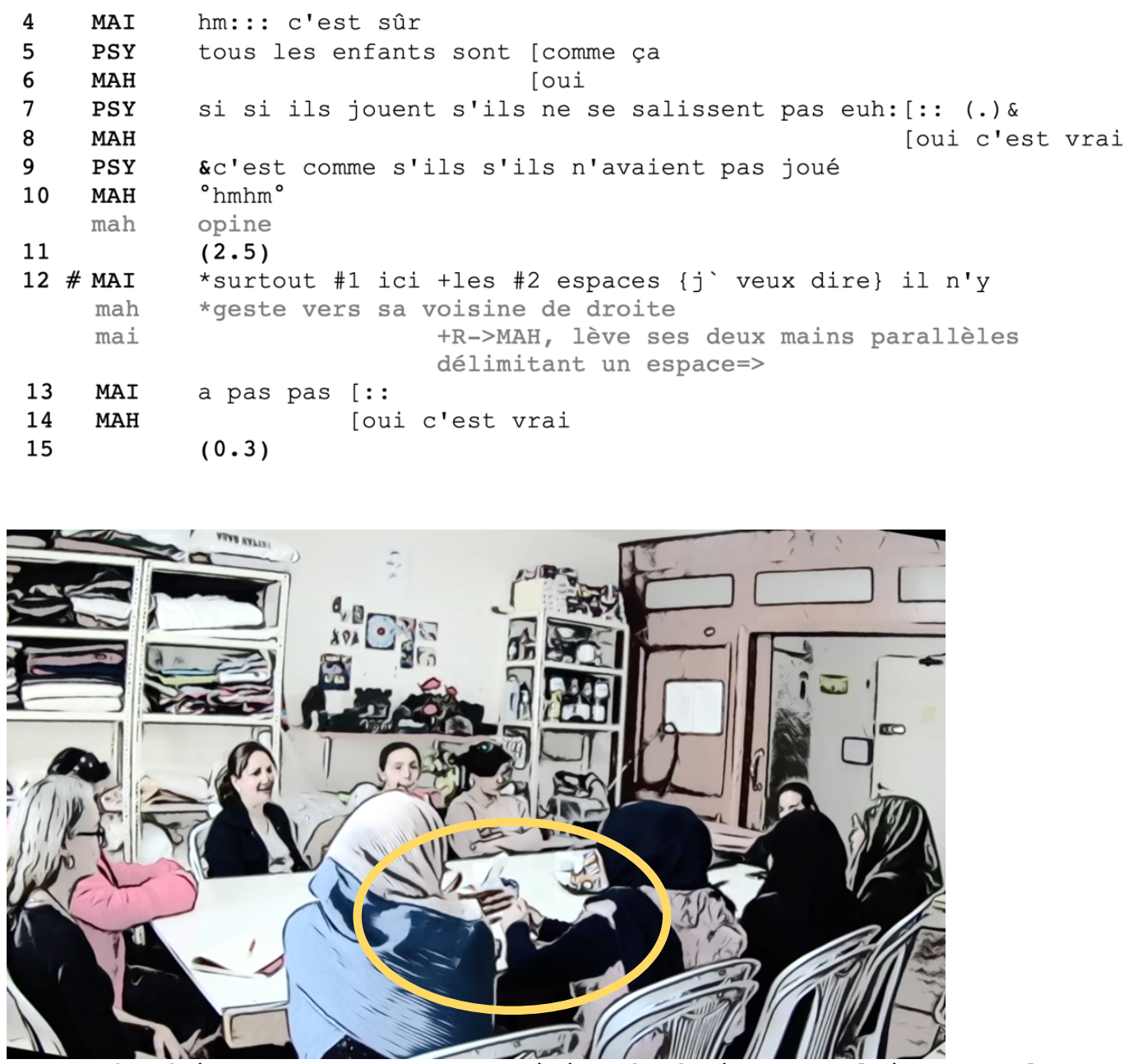

Im1. Maha fait un geste vers sa voisine de droite pour lui passer le tour

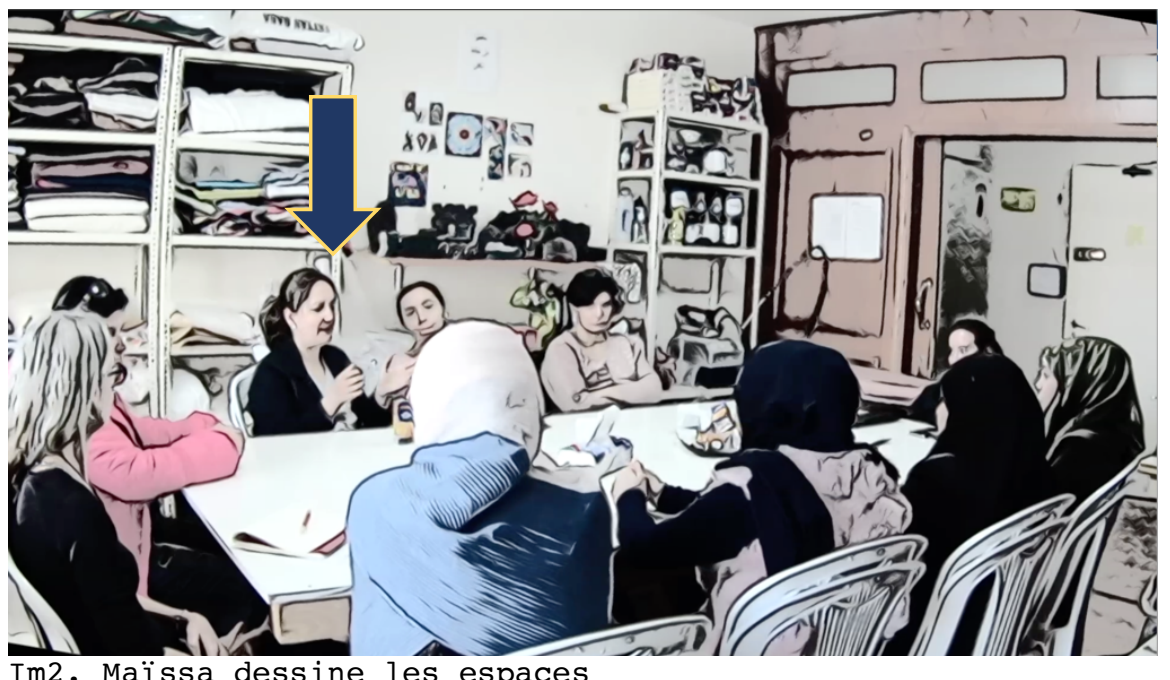




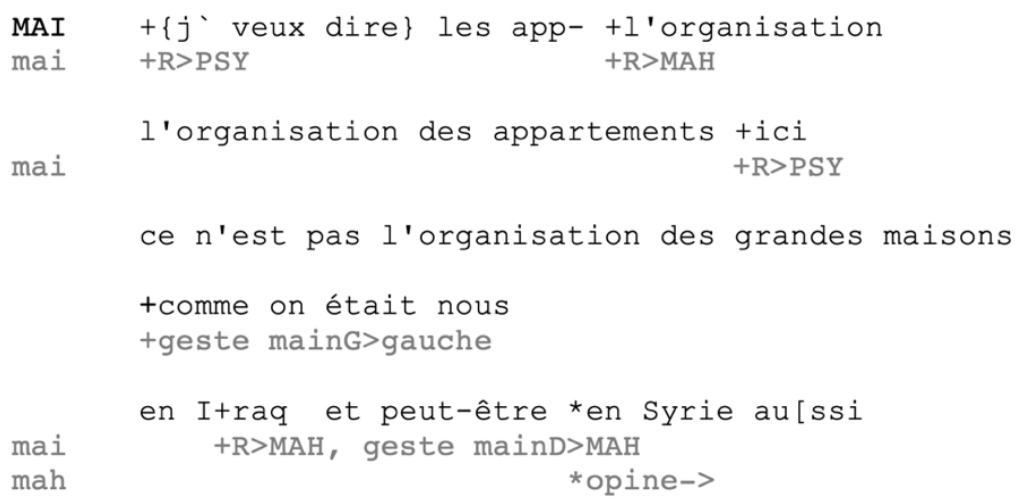

[oui aus $\left[\mathrm{s}^{*} \mathrm{i}+\right.$

$+\mathrm{R}>\mathrm{PSY}$

Sur le plan séquentiel, l'intervention de Maïssa à la ligne 12 modifie le déroulement prévisible du tour de table, puisqu'elle se trouve en face, et non à côté de Maha, et qu'elle n'a pas été désignée par cette dernière comme la prochaine locutrice (image 1). En même temps, elle commence son tour de parole par "surtout ici" (xaștan hūn), le présentant donc comme une expansion d'une parole précédente. Déterminer un tour de parole auquel le tour de Maïssa se raccrocherait sur le plan syntaxique reste difficile, mais, sur le plan sémantique, le lien se fait sans hésitation avec le tour de Maha (1-3) puisque Maïssa vient confirmer la difficulté à tenir ses enfants propres, en considérant cette difficulté comme particulièrement liée à "ici". Les lignes 12 à 20 constituent une introduction au récit de Maïssa, qui ne présente que certaines des caractéristiques des préfaces (Sacks 1992). En particulier, elle ne vise pas à suspendre l'alternance des tours de parole (puisque l'organisation de la parole est déjà différente de celle d'une conversation). En revanche, elle permet de mettre en place plusieurs conditions favorables au développement du récit.

La première est la réorganisation du cadre de participation. Le moment où Maïssa prend la parole est un moment de transition dans le tour de table. L'emplacement séquentiel qu'elle choisit et la façon dont elle débute son tour de parole indiquent qu'elle s'inscrit en continuité avec le tour de Maha. On observe d'ailleurs que Maha s'aligne avec le tour de Maïssa et s'affilie immédiatement à ses propos (ligne 14). Maïssa modifie donc l'organisation de la participation à un premier niveau, celui de l'espace interlocutif (locuteur / récepteur). De réceptrice, elle se positionne comme locutrice additionnelle ou co-locutrice en soutien d'une autre parole. Dans la suite de son tour, elle modifie aussi l'organisation du groupe. L'organisation collective en place jusque-là reposait sur (au moins) deux dimensions identitaires. Une première organisation était liée à l'interlocution, avec une locutrice sur la sellette (occupant le floor) et un auditoire, au sein duquel la psychologue occupait une place particulière, étant celle qui avait attribué la parole, celle vers qui les regards de la locutrice étaient orientés plus de la moitié du temps, et celle qui évaluait la prise de parole en cours comme suffisante (complète) ou non. Une autre organisation du groupe était liée à la formation suivie par les femmes (aghabâni ou broderie manuelle), les deux groupes étant installés de part et d'autre de la table. Maïssa ajoute une autre dimension d'organisation du collectif par le recours à l'opposition "ici, ailleurs" (17-18). Elle commence par mentionner les espaces (ligne 12), puis reformule en parlant de l'organisation des logements (lignes 16-17). Les deux formulations utilisent une construction syntaxique proche "les espaces $\{j$ veux dire $\}$ il n'y a pas" (énoncé inachevé) -> 
"I'organisation l'organisation des logements ici (.) ce n'est pas...", qui exprime un manque, une absence, et qui projette la deuxième partie d'une construction binaire (qui concernerait à la fois l'ailleurs et le versant positif de l'assertion). Puis elle poursuit en opposant (17) "I'organisation des appartements ici" (niợām effiqāq hūn) à "I'organisation des grandes maisons comme on était en Iraq" (niợām elbjūt lekbīr mitl ma kanna bi I ఢirāq, 17-20).

Cette opposition est aussi marquée par un geste évoquant (ligne 17 ci-dessous) le caractère clos des petits logements au Liban - si le terme d'appartement n'est pas formulé, il est incarné par l'espace cloisonné et délimité par les deux mains raidies de la locutrice, image $3 a, 3 b-$ contrastant avec le geste ample et circulaire dessinant de grandes maisons en Iraq (ligne 8 cidessous, images $4 a, b, c, d, e)$ :

Extrait 5 (reprise extrait 4)

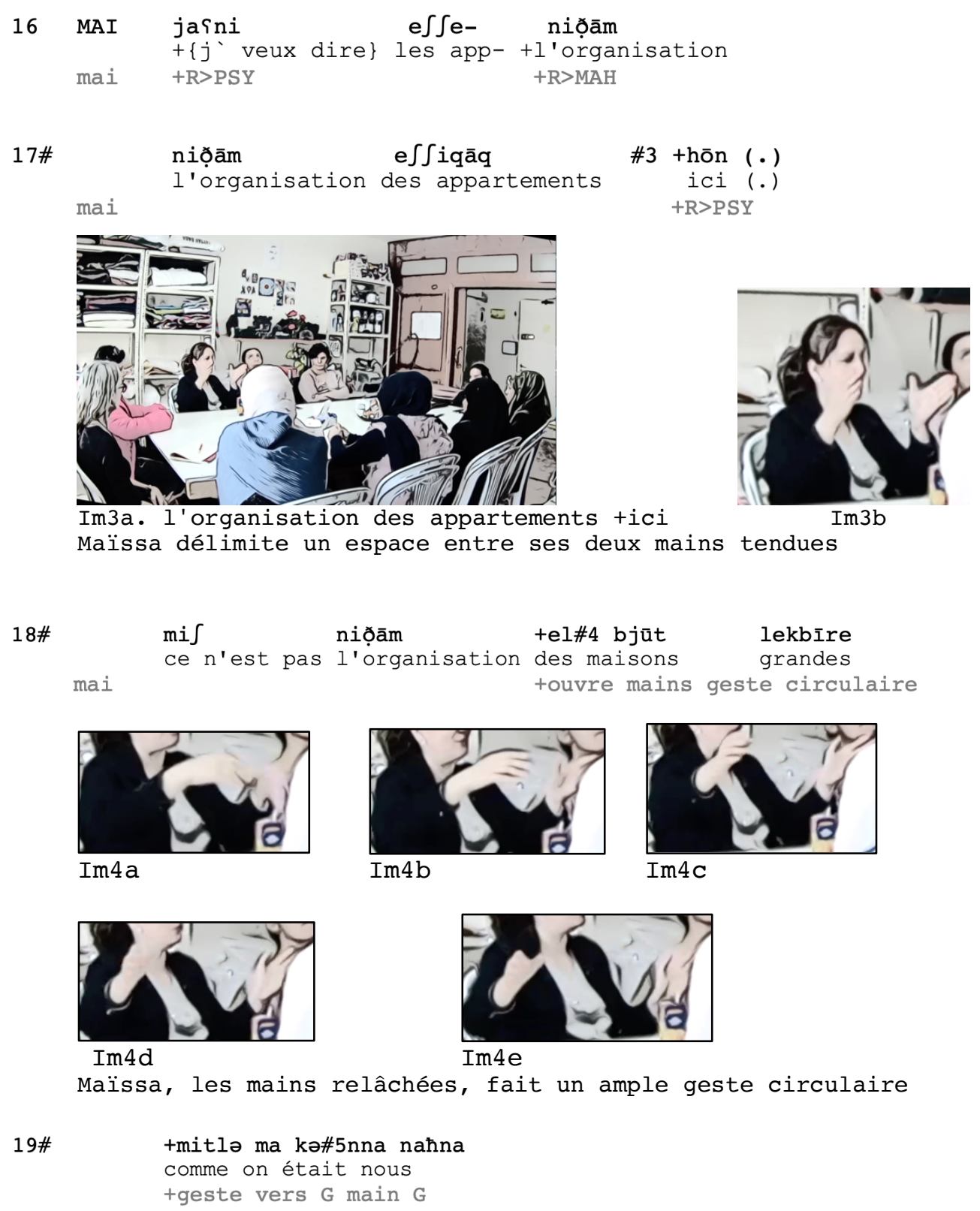



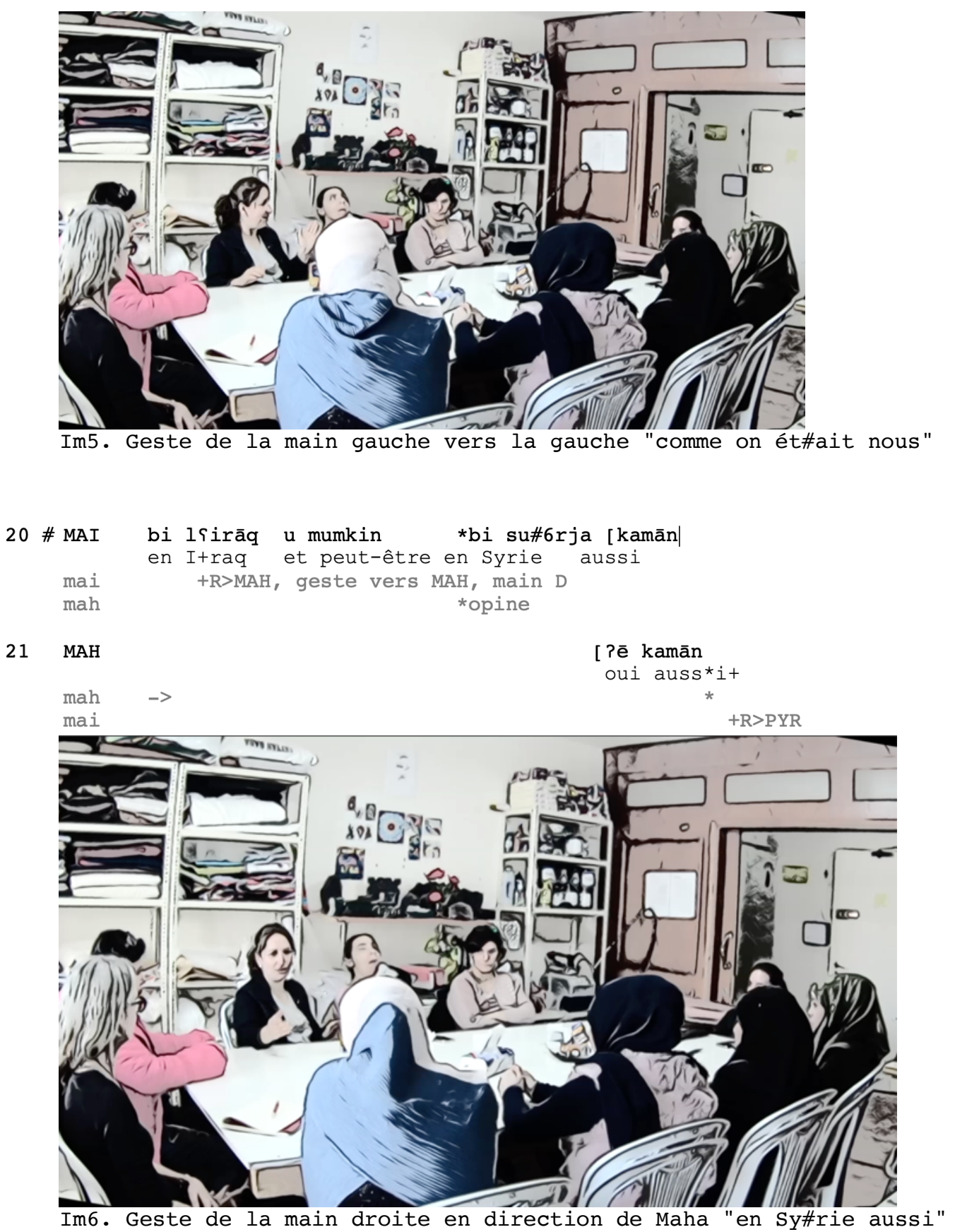

Cette partie du tour de Maïssa conduit à établir un possible point commun (un attribut partagé) entre la locutrice (Iraquienne) et les participantes syriennes ("et peut-être en Syrie aussi"). Cette proposition suscite non seulement l'accord de Maha (qui opine de façon régulière), mais aussi celui de plusieurs des femmes (syriennes), assises face à Maïssa, qui opinent.

A la suite de cette introduction, Maïssa commence le récit de la vie d'avant (extrait 6) en parlant, non plus comme une voix en soutien du tour de Maha, mais au nom d'un "nous" qui partage une expérience commune. Elle l'indique par l'emploi du pronom "nous" ("nous nos maisons elles étaient euh:", "naћna bjūtna kānet Ø:::"), dans une construction avec le pronom disjoint "naћna" (nous) en début de tour (que l'on peut rendre par une construction 
disloquée ${ }^{14}$ en français), qui renforce l'opposition entre les maisons d'Iraq (et possiblement de Syrie) et celles du Liban ${ }^{15}$. La locutrice enchaîne, le regard orienté vers la psychologue, en décrivant comment étaient les maisons "là-bas" :

Extrait 6

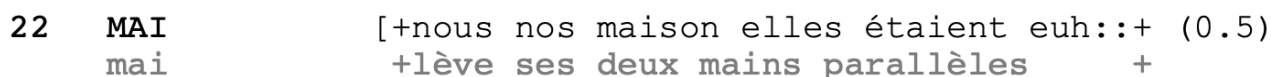

$23+\{j$ veux dire $\}$ des constructions horizontales

mai +mains position verticale, maind balaie maing

24 +pas verticales

mai +mains parallèles, mvt vers le haut

$(0.4)$

25 PYR oui

26 MAI $+\{j$ 'veux dire $\}$ une maison à côté de l'autre tet il y avait mai +geste maind marquant des places successives +geste séparation

27 une clôture (0.5) tet tu avais une grande maison et t tu mai +geste 2 mains vers 1 'avant +bis

$28 \#$ avais un jardin et ttu avais un garage (.) \{alors\} \#8 mai + bis

$29 \# \begin{aligned} & \text { +l'enfant était plus à son \#9 } \# \mathbb{I}[\text { aise pour jouer } \\ & \text { mai +bis }\end{aligned}$

$30 \quad \mathrm{MAH}$

mah

sam

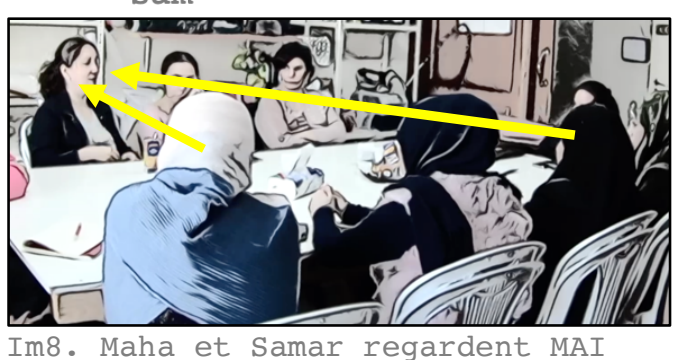

Im8. Maha et Samar regardent MAI

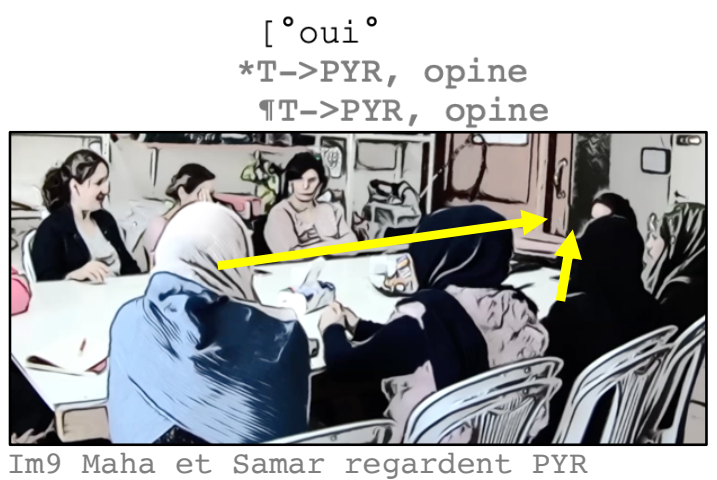

Im9 Maha et Samar regardent PYR
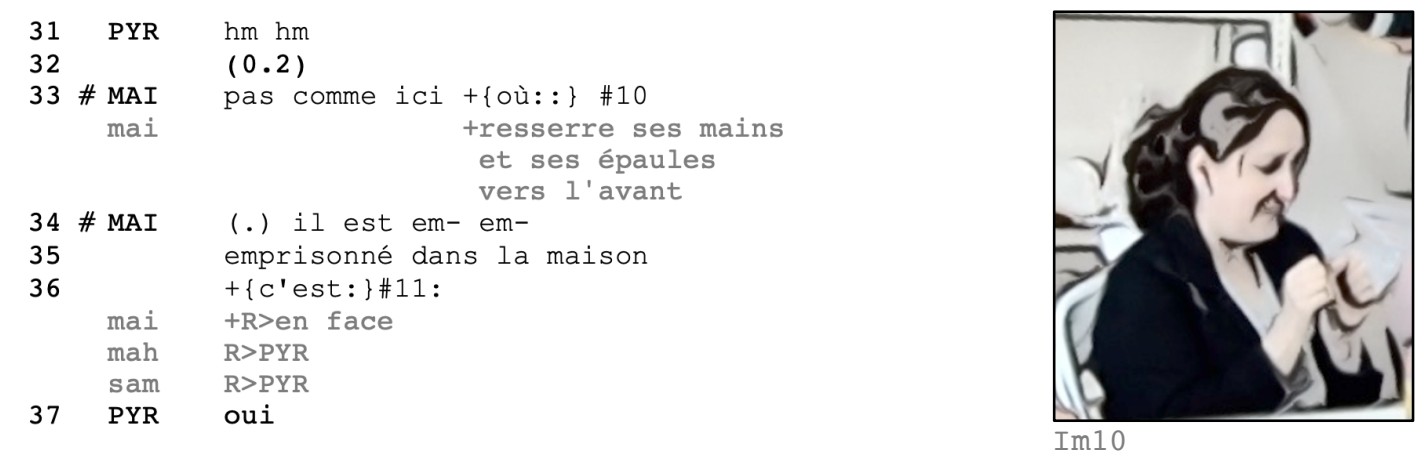

\footnotetext{
${ }^{14}$ Un.une relecteur.trice anonyme me signale qu'il s'agit d'un nominativus pendens plus que d'une dislocation à gauche.

${ }^{15}$ Sur la fonction contrastive des constructions disloquées, voir Pekarek Doehler, De Stefani \& Horlacher 2015.
} 


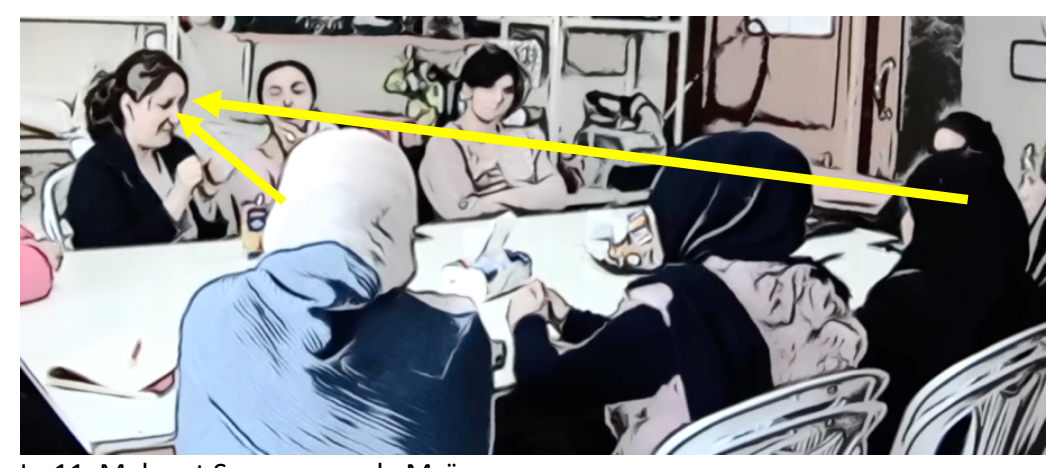

Im11. Maha et Samar regarde Maïssa

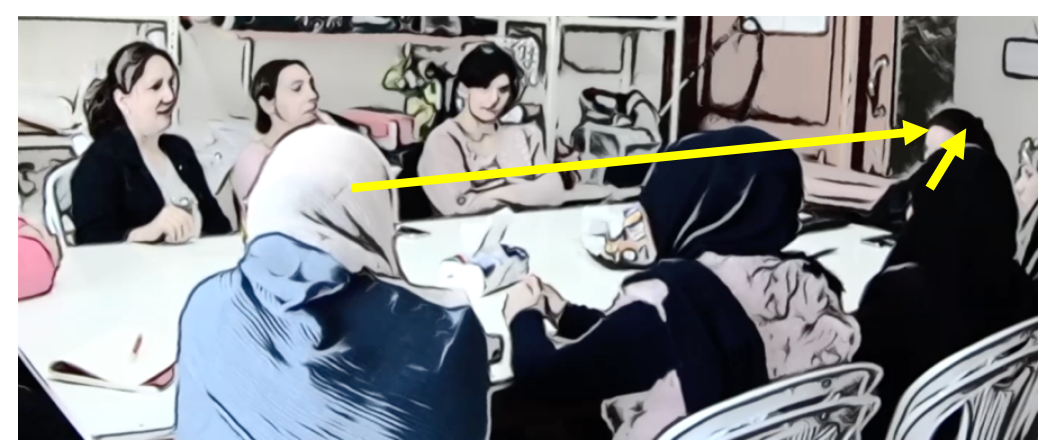

Im12. Maha et Samar ont tourné la tête vers la psychologue, alors que Maïssa regarde dans leur direction

Après une description des maisons en Iraq, lignes 22-28, Maïssa explicite, à la ligne 29, le lien avec les enfants dont parlait Maha. Enfin, à la ligne 33, elle établit un contraste avec ce qui se passe "ici" ("pas comme ici", "mif mital hūn"), où l'enfant "est emprisonné dans la maison"

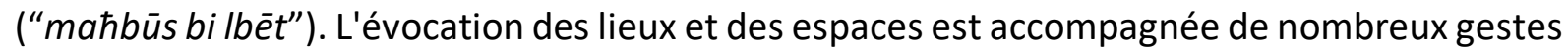
qui renforcent les contrastes entre les grands espaces (avec leurs composantes, cf. les gestes bâtons lignes 27-28) et les espaces qui emprisonnent (rétraction des bras et des épaules, 33, images 10, 11).

La construction de l'interlocution à travers les regards est aussi intéressante. Une première fois, à la ligne 30, deux membres du groupe (Maha et Samar) expriment corporellement leur affiliation aux propos de Maïssa (elles opinent de la tête), et elles soulignent la structuration du récit en cours : sur l'énoncé "alors l'enfant était plus à son aise pour jouer", qui apparaît comme une sorte de conclusion de ce qui précède, et une possible fin du tour de Maïssa (ou au moins une articulation forte), elles s'orientent vers la psychologue, manifestant leur attente de sa réaction. La même configuration se produit à nouveau à la ligne 36 , à la fin du tour de Maïssa, qui est laissé en suspens par la locutrice, alors qu'elle-même regarde vers les femmes en face d'elles, Maha et Samar tournent la tête vers la psychologue, manifestant à nouveau l'attente d'une réaction. A la ligne 37, la psychologue produit un simple accusé de réception, indiquant ainsi qu'elle ne reprend pas le tour, ce qui conduit Maïssa à poursuivre. Les contributions essentiellement silencieuses (hormis le "oui" très bas de Maha ligne 30) et gestuelles ou posturales de Maha et Samar incarnent elles aussi I'organisation sociointeractionnelle mise en place par Maïssa : ces deux participantes agissent comme parties actives du "nous". L'affiliation entre les participantes mettant en place une forme de locutrice collective portant la parole de celles qui ont quitté leur pays s'exprime encore à la fin du tour de Maïssa : 
Extrait 7

$38 \quad$ MAI

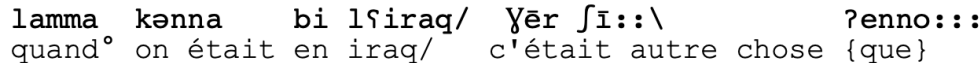

39 hajdi lə3nejne u bafrif

Ponno * 4 am jalৎabu *u hēk
40 que *ils jouent fet tout

40 mah que *ils jouent $\begin{aligned} & \text { (et tout ça } \\ & \text { *opine }\end{aligned}$

41 PSY Pe

oui

42

$(0.5)$

43 MAI

bass hūn Penno: : : (.) b Jūfha

sạb ?enno lwalad

mais ici \{que\} (.) je trouve ça difficile que l'enfant

44

jṭilas ma btårfi wēn rājeh (0.4) u: : : (0.3)

sorte tu ne sais pas où il va $(0.4)$ et:: : $(0.3)$

45

bi hal ca3?a uSawāris u: : (0.9)

dans cette agitation et ces rues et: : (0.9)

KTİR ȘA B jåni

C'est TRES DIFFICILE \{en fait\}

47 MAH $\quad[\mathrm{hm}$

48 MAI

mai

mai

49 MAH

50

MAH
MA

51

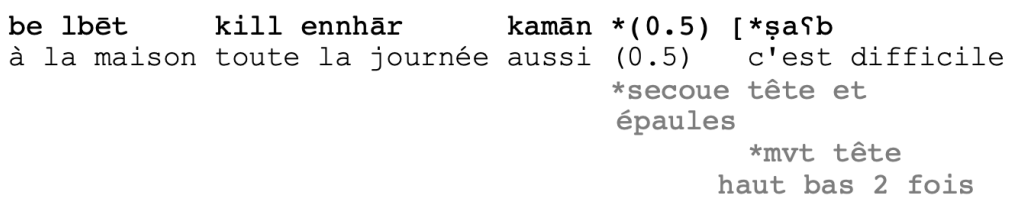

$(0.5)$

Je n'entrerai pas dans une analyse détaillée de l'extrait, mais soulignerai simplement deux éléments.

D'une part, on voit que Maïssa construit cette dernière partie de son intervention à nouveau à partir d'un contraste ("quand on était en Iraq..." 38, "mais ici" 43), soulignant les difficultés à Beyrouth et la tranquillité en Iraq.

A la ligne 49, en chevauchement avec l'évaluation finale de Maïssa, Maha prend la parole pour énoncer à son tour une évaluation, venant ainsi entériner la clôture du récit. Cet ajout est particulièrement intéressant à observer à la lumière des processus de reprises, transformation et recyclage de ressources déjà présentes dans l'interaction. II reproduit tout d'abord la

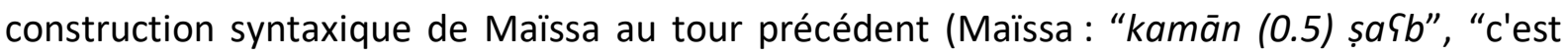

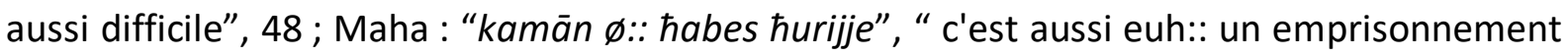
de la liberté", 49). II reprend aussi le même type de construction que dans l'initiation du tour 
de Maïssa ligne 12, extrait 5, "xaștan", "surtout" et "kamān", "aussi" (des expansions adverbiales) et la même modalité de complétion du tour. Enfin, la locutrice recycle et

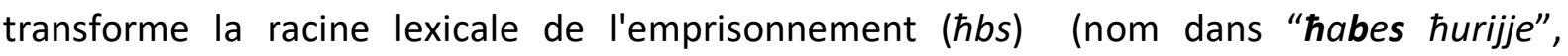

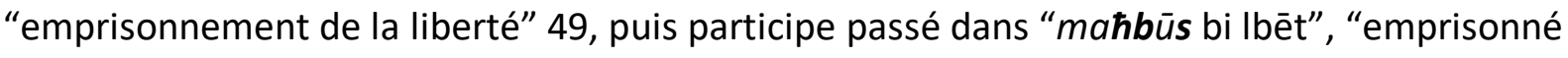
dans la maison", employé par Maïssa ligne 35, extrait 6).

Le tour de parole de Maha entraîne un bref échange affiliatif entre elle et Maïssa, qui vient achever la clôture (Traverso 2005) : ligne 49, Maïssa, qui regardait la psychologue, se tourne vers Maha, puis, à la ligne 51, elle confirme (avec un "oui" et une inclinaison de la tête), avant d'énoncer une nouvelle fois sa propre évaluation, intégrant ainsi l'apport de Maïssa dans son propre discours, pendant que Maha elle aussi apporte une ratification (ligne 52). L'une et l'autre marquent alors la fin de l'échange, en détournant leurs regards réciproques, Maha se tournant vers la psychologue, alors que Maïssa regarde vers le haut.

En résumé, on peut voir la prise de parole à partir de la ligne 22, comme émanant - à travers la voix de Maïssa - d'une locutrice collective, qui fait un récit de la vie d'avant, des ressentis et de la position des femmes réfugiées du groupe, accompagné, soutenu et complété par les contributions (verbales, vocales ou gestuelles) des autres participantes. Dans ce récit sont mis en oeuvre de nombreux procédés de reprise, de transformation et d'écho sur le plan verbal et de "corporalisation" des positions socio-interactionnelles. Le récit, et d'ailleurs la prise de parole qui le précède à propos des enfants, font momentanément dévier le sujet initial qui était de préciser ce qu'est la propreté pour chacune. Il modifie aussi l'organisation à l'intérieur du groupe. Par rapport aux attributions identitaires faites par la situation et l'activité, il déplace les échanges vers des considérations générales et partagées avec les autres femmes réfugiées du groupe concernant la vie d'autrefois, plus nantie - énumération des biens possédés, lignes 27-28 - et plus confortable ("raha, "aise , confort", ligne 29), par rapport à la vie d'aujourd'hui, ici. Il permet de la sorte de référer à d'autres traits identitaires que "femmes nécessitant des séances de Life skills ou femmes en besoin de soutien auprès des associations d'aide aux réfugiés".

\subsection{Raconter pour se distinguer : le savon d'Alep au laurier}

Le deuxième extrait, que j'examinerai plus succinctement, comporte un récit d'avant qui met en place des constructions socio-interlocutives assez différentes, et sans doute plus complexes, au sein du groupe ${ }^{16}$. Il fait aussi intervenir d'autres ressources. La formatrice du groupe d'aghabâni, Monira, s'est jointe à la séance pendant le tour de table. Une fois que chacune des bénéficiaires a exprimé son point de vue sur l'hygiène, la psychologue lui donne la parole, et elle se lance alors dans un long plaidoyer sur l'importance de l'hygiène, expliquant notamment qu'une mauvaise hygiène crée une forme de fatigue. Maha (la même participante que dans l'extrait précédent) enchaîne alors avec un premier récit, à propos d'une grippe, qui va conduire Monira à enchaîner avec un récit d'avant. Observons pour commencer le récit de la grippe :

\section{Extrait 8}

\footnotetext{
${ }^{16}$ Pour une étude de la mise en place d'identités, de positionnements et de distinction dans une réunion au départ "dichotomique", voir Traverso 2017.
} 


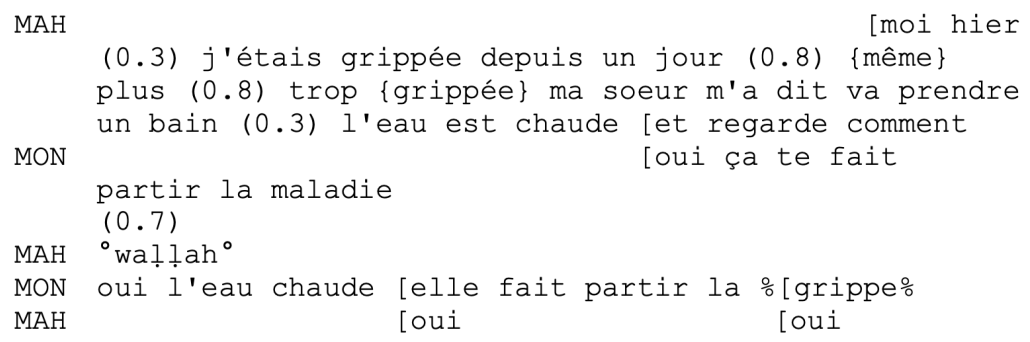

Le petit récit de Maha, de facture classique ${ }^{17}$, est intéressant au niveau des positionnements et des affiliations. Il intervient en illustration des propos antérieurs de Monira sur l'énergie de l'eau et ses vertus thérapeutiques. A la fin de ce récit, lignes 10 à 12 , ces deux participantes opèrent à deux voix la transition vers la reprise de l'interaction tour à tour : Monira, aux lignes 7-8, énonce une formule générale (Holt \& Drew 1998, Traverso 2005) ("ça te fait partir la maladie"), et Maha ligne 10 confirme à l'aide de la particule discursive "wallah" (Traverso 2002), puis Monira clôt le récit ligne 11, par une reprise de son énoncé précédent sous format disloqué à gauche ("oui oui l'eau chaude elle fait partir la \%grippe\%). Après quelques tours peu audibles, Monira introduit son récit d'avant :

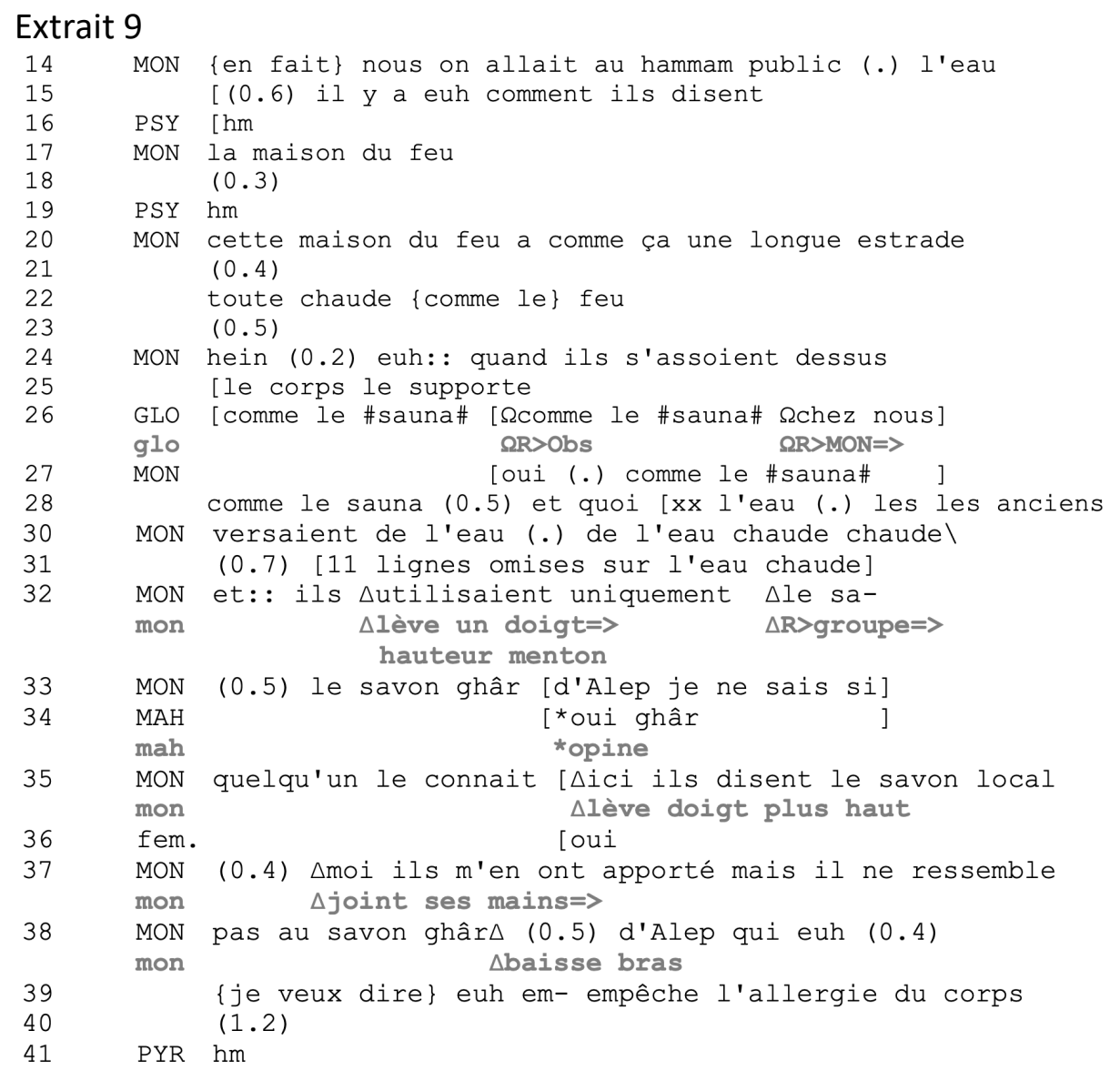

Monira développe son récit sur les vertus du hammam en regardant la psychologue, pendant que cette dernière produit des continueurs $(16,19)$. L'intervention de Gloria (I. 26) vient momentanément interrompre le face-à-face entre Monira et la psychologue, et tout le monde

\footnotetext{
17 Il se conforme en effet aux canons du genre narratif, avec un déplacement vers un autre temps précis (hier), une organisation temporelle qui soutient une mise en intrigue, laquelle mène à une résolution.
} 
se focalise sur elle. Elle compare le hammam au sauna, ce qui peut aussi laisser entendre la mise en contraste d'une réalité occidentale et moderne (le sauna "chez nous") à une autre, orientale et traditionnelle, dont est en train de parler Monira ${ }^{18}$. L'énoncé est produit en chevauchement sur le tour de Monira, qui répète cette proposition à la ligne 27, puis une nouvelle fois après le chevauchement à la ligne 28.

Dans la suite du récit, Monira parle du savon artisanal (le savon au laurier, șabun Xār) emblématique de sa ville, Alep. Aux lignes 32-33, elle le met en relief dans son tour de parole par un ensemble de procédés : gestuelle du doigt levé, regard qui parcourt le groupe, autoréparation intégrant une pause relativement longue avant de prononcer le nom. Puis, aux lignes 33-35, avec "je ne sais pas si quelqu'un connaît", elle présente la réalité dont elle parle comme pouvant être inconnue des autres participantes, y compris des femmes syriennes du groupe. Elle construit ainsi le groupe bien différemment de ce qu'a fait Maïssa dans le récit précédent, qui a mis en place une voix collective (des réfugiées ou des Syriennes), qu'elle a ensuite fait entendre. Ici, non seulement Monira implicite que les autres participantes pourraient ne pas savoir ce dont elle parle, mais lorsque ces dernières manifestent au contraire qu'elles connaissent le savon d'Alep (ligne 36, la plupart des femmes du groupe, où qu'elles soient assises autour de la table et qu'elles soient ou non réfugiées opinent fortement), elle enchaîne en attirant l'attention sur le fait que le savon au laurier d'Alep est bien différent (et bien plus hygiénique) que le savon local libanais. Dans sa prise de parole, Monira opère ainsi des ligne de distinction, plus complexes que ce que Maïssa a fait dans son récit, qui ne se résument pas "ici"/“là-bas"19.

En revanche, comme l'a fait Maïssa, elle mentionne les différences du savon au laurier d'Alep avec le savon artisanal libanais (lignes 35 à 38), en soulignant que ce dernier ne présente pas les qualités aseptisantes du savon ghâr et met en avant un contraste (marqué notamment par l'opposition "je" vs "ils").

\subsection{La réception des récits}

Le récit de Monira, comme celui de Maïssa, est un récit qui fait valoir des habitudes d'avant et qui évoque ce qui était mieux dans le pays quitté par rapport à ce qui a cours ici. Dans ces deux récits, le pôle négatif de la comparaison est simplement désigné par "ici" (hūn), et face au "nous" ou au "je", on entend seulement "ils". Le Liban, Beyrouth ou les Libanais ne sont pas explicitement mentionnés. Dans ces récits, ce ne sont ni les protagonistes (voir par exemple le flou de la référence du pronom "ils" dans le récit de Monira, "ils m'en ont apporté"), ni les faits précis qui importent, mais l'évocation fugace d'un autre temps et la mise en exergue de différences quant aux manières de faire et de vivre, assorties d'un jugement de valeur sousjacent à travers lequel se laissent percevoir toutes les difficultés de la condition de réfugié. Je me suis concentrée sur l'introduction et la réception des récit entre les personnes qui s'y trouvent liées (ayant produit le tour qui le suscite, ou s'y engageant par des continueurs, des marques d'accord, verbales ou gestuelles, des évaluations ou en collaborant à leur clôture).

\footnotetext{
${ }^{18}$ Sur les représentations contrastées du hammam au Moyen-Orient telles qu'elles s'expriment dans des réunions, voir Traverso 2014, 2017.

${ }^{19}$ On peut avancer l'hypothèse qu'en opérant ces distinctions, Monira travaille à éviter d'apparaître comme "pair" des autres femmes (bien qu'elle soit aussi réfugiée syrienne, elle n'est pas bénéficiaire, mais salariée de l'association). Sur la question des pairs et des tiers, voir le Carnet de recherche Tiasamn, https://tiasamn.hypotheses.org.
} 
Mais au-delà de ces moments, l'espace que ces récits ouvrent dans l'économie générale des séances est bien vite refermé dans les enchaînement séquentiels. Chacun d'eux suscite en effet une réaction d'une des femmes libanaises du groupe. Après le récit de Maïssa, une femme réfute la pertinence de ce que le récit a illustré, en argumentant que les virus s'attrapent aussi à la maison et que donc " ça n'a pas de rapport que tu les prives de liberté ", puis en enchaînant longuement sur toutes les erreurs que l'on commet sur le plan de l'hygiène dans les maisons. Elle réfute ainsi la pertinence de ce qui vient d'être dit et réoriente la discussion sur le thème de l'hygiène en général.

Suite au récit de Monira, la même participante prend la parole pour se moquer des pratiques anciennes du hammam où "les grand-mères t'ébouillantaient", et elle les met en cause en rappelant que les médecins préconisent aujourd'hui au contraire de ne pas agresser la peau et de se laver avec des produits doux.

Ces interventions opèrent des recadrages. Elles extraient un sens du récit ("au Liban, il faut priver les enfants de liberté pour qu'ils restent propres", "le hammam était une pratique hygiénique et le savon d'Alep est aseptisant") qu'elles traitent comme inexact.

La psychologue, elle, si elle écoute toutes les prises de parole sans interrompre ni recadrer, produit des continueurs, voire des marques d'accord, ne reprend pas ces récits d'avant, qui apparaissent donc dans le déroulement des séances, comme des moments, récurrents mais fugaces.

\section{Conclusion}

Les récits d'avant sont très éloignés des récits canoniques ou même de véritables chroniques de la vie d'avant. Ils décrochent vers un temps passé, mais ne sont pas organisés sous forme d'une suite d'événements; ils évoquent un temps qui ne se déroule pas; ils rappellent sous forme de fragments des manières perdues de vivre et de faire.

Par ces récits, les femmes mettent en scène des instances, individuelles ou collectives, prenant ou donnant voix dans le collectif. II peut arriver qu'elles argumentent et remettent clairement en question le bien-fondé de ce qui leur est dit (Traverso 2019, Kerbage \& Traverso 2020, Traverso \& Kerbage 2020), mais c'est plutôt rare.

La question qui se pose au final est celle de l'impact de ces récits et des transformations locales qu'ils opèrent sur les séances, leurs attendus et leurs principes, sur les thèmes ou sur le groupe. A un premier niveau, l'analyse des récits a montré que, dans le déroulement séquentiel de l'interaction, ils sont suivis d'un retour vers le cadre (sujet, format) posé au départ. Les récits sont fugitifs, et l'espace qu'ils ouvrent fragile et fugitif. Plus globalement, le terrain d'une année effectué dans cette association n'a pas fait apparaître de changement sensible des activités de Life Skills, en termes de thème ou d'activité, sinon les modifications liées aux nouvelles exigences et directives imposées par les bailleurs. Ces récits d'avant sont pourtant récurrents et ils émergent séance après séance, à différentes occasions, pour exprimer par contraste les manques et les difficultés de la vie "ici" et rappeler des aspects de la vie d'autrefois. Leur récurrence, et les réparties qu'ils obtiennent le plus souvent de la part des animatrices ou des bénéficiaires libanaises, me semblent en montrer deux dimensions, que je mettrai en lien avec l'activité même de "soutien psychosocial pour les personnes réfugiées et précaires" et ce qu'elle recèle de contrainte, d'attribution d'image et de proposition/imposition de principes, souvent inapplicables dans les conditions où les personnes vivent (Kerbage \& Traverso 2020). Dans les entretiens, les intervenantes, des jeunes psychologues, parfois encore en formation, ou des travailleuses sociales expriment souvent leur malaise et la difficulté de leur tâche (Traverso 2019). Elles ont bien conscience 
que les bénéficiaires assistent aux séances pour des raisons très diverses, allant de la occasion de sortir de chez elles, à apprendre quelque chose, rencontrer d'autres personnes, avoir leurs enfants pris en charge pour des activités collectives pendant leurs propres heures de formation, etc. Dans la pratique, beaucoup de ces femmes attendent simplement que ça passe et parfois bavardent entre elles, certaines discutent sur les thèmes abordés, certaines contestent ce qui est dit, certaines racontent. C'est dans ce contexte qu'il faut envisager ce qu'elles font par ces récits d'avant.

L'analyse dans la lignée de Goodwin, avec la prise en compte des ressources diversifiées, des corps des participantes et de la mise en place d'instances collectives incarnées a montré que ces récits activent dans le groupe un autre collectif, qui, à travers le récit, développe une pratique de résistance à l'activité et à ce qu'elle met en place. Ceci se joue et se rejoue de façon répétitive, et suscite, de façon tout aussi récurrente, des réparties, des recadrages, voire des remises en place (comme "ça n'a rien à voir que tu le prives de liberté") ${ }^{20}$, en ce sens on pourrait parler d'un jeu de chicane.

Parallèlement, les récits représentent aussi des échappées narratives, qui entraînent brièvement en dehors de la situation locale (la séance de Life skills) ou plus générale (personne déplacée). A travers leur production, les femmes évoquent, se rappellent et font valoir collectivement, des habitudes, comme un pied-de-nez aux contraintes et aux difficultés, mais aussi comme une façon de s'approprier, à de brefs moments, le temps d'échange de la séance censée favoriser le bien-être psychosocial.

\section{Références}

Antaki C., Widdicombe S. (1998). Identities in talk. London: Sage Publications.

Bamberg M., Georgakopoulou A. (2008), Small Stories as a New Perspective in Narrative and Identity Analysis, Text \& Talk, 28, 377-396.

Bercelli F., Rossano F., Viaro M. (2008). Different place, different action: Clients' personal narratives in psychotherapy. Text \& Talk 28, 283-305.

Bonu B. (2001). Les évaluations conversationnelles dans la narration. Revue québécoise de linguistique 29, 1, 50-69.

Bres, J. (2001). De la textualité narrative en récit oral : l'enchaînement des propositions. Revue québécoise de linguistique, 29 (1), 23-49.

Canagarajah S. (2017). The Routledge Handbook of Migration and Language. Londres : Routledge.

Chambon N. (2018) Raconter son histoire comme personne : les migrants et leurs récits. Le sujet dans la Cité 9 (2), 63.

Chambon N. \& Traverso V. (dir) (à paraître), Raconter, relater, traduire : paroles de la migration, Lambert Lucas.

De Fina A., Tseng A. (2017). Narrative in the study of migrants. In Canagarajah S. The Routledge Handbook of Migration and Language, 381-396.

D'Halluin E., (2012). Les épreuves de l'asile. Paris : Editions de l'EHESS.

Drew P., Holt E. (1998). Figures of speech: Idiomatic expressions and the management of topic transition in conversation. Language in Society, 27, 4, 495-522.

Filliettaz L., Revaz F. (2006). Actualités du récit dans le champ de la linguistique des discours oraux. Le cas des narrations en situation d'entretien. Protée 32, 2-3, 53-66.

Georgakopoulou A. (2006). The other side of the story: Towards a narrative analysis of narratives-ininteraction. Discourse studies, 8, 2, 265-285.

Georgakopoulou A., De Fina A. (ed.) (2015). Handbook of Narrative Analysis. Wiley Blackwell.

Goffman E. (1981), Forms of Talk, Oxford, Blackwell.

\footnotetext{
${ }^{20}$ En écho aux résultats obtenus par Marranconi \& Kerbage 2017 sur les images stéréotypées des réfugiés syriens parmi les soignants et travailleurs sociaux qui travaillent avec eux, on pourrait aussi faire l'hypothèse que ce jeu d'agacement prend aussi sens dans le contexte complexe, et souvent difficile, des relations entre les déplacés syriens et les Libanais.
} 
Goodwin C. (1984), "Notes on story structure and the organization of participation”, in Atkinson J., Heritage J. (eds), Structures of Social Action: Studies in Conversation Analysi., London : CUP, 225-246.

Goodwin C. (1986), "Between and within: Alternative sequential treatments of continuers and assessments", Human Studies 9(2-3), 205-217.

Goodwin C. (1997). The blackness of black. In Resnick L. B., Salijo R., Pontecorvo C., Burge B. (eds), Discourse, Tools and Reasoning. Essays on Situated Cognition. Berlin, Springer, p. 111-140.

Goodwin C. (2004), “A Competent Speaker Who Can't Speak: the Social Life of Aphasia”, Journal of Linguistic Anthropology, 14(2), p. 151-170.

Goodwin C. (2007). Interactive footing. In Holt E. \& Clift R. (eds), Reporting Talk: Reported Speech in Interaction, Cambridge, Cambridge University Press, 16-46.

Goodwin C. (2013). The co-operative, transformative organization of human action and knowledge, Journal of Pragmatics 46 (1), 8-23.

Goodwin C. (2015). Narrative as talk-in-interaction. In De Fina A. \& Georgakopoulou A. (eds), The Handbook of Narrative Analysis, London, John Wiley \& Sons, 195-218.

Goodwin C. (2018). Co-operative action, Cambridge : CUP.

Goodwin C., Goodwin M., Olsher D. (2002). Producing Sense with Nonsense Syllables: Turn and Sequence in Conversations with a Man with Severe Aphasia. In Ford C., Fox B., Thompson S. (eds), The Language of Turn and Sequence, Oxford, Oxford University Press, 56-80.

Goodwin M. (1982). 'Instigating': Storytelling as Social Process. American Ethnologist 9(4), 799-819.

Goodwin C., Goodwin M. (1987). Concurrent Operations on Talk: Notes on the Interactive Organization of Assessments. Pragmatics 1, 1-54.

Greco L., Mondada L., Renaud P. (dir). Identités en interaction, Limoges, Lambert Lucas.

Groupe ARAPI (2019). Corpus d'arabe parlé (4) La convention de transcription ARAPI pour l'arabe parlé en interaction, Carnet de I'Ifpo, https://ifpo.hypotheses.org/9335.

Inter-Agency Standing Committee. (2007). Guidelines on mental health and psychosocial support in emergency settings.

http://www.who.int/mental_health/emergencies/guidelines_iasc_mental_health_psychosocial_june_2 007.pdf.

Jefferson G. (1978). Sequential aspects of storytelling in conversation. In J. Schenkein (Ed.) Studies in the organization of conversational interaction, New York : Academic Press, 219-248.

Jefferson G. (1988). On the sequential Organization of Troubles-Talk in Ordinary Conversation. Social Problems $35(4), 418-440$.

Kerbage H.,\& Traverso V. (2020). Life skills et awareness sessions (1) : le thème de I'hygiène. Carnet de l'Ifpo. https://ifpo.hypotheses.org/

Labov, W., Waletsky, J. (1967). Narrative analysis: Oral versions of personal experiences. In J. Helm (Ed.), Essays on the verbal and visual arts. Seattle: University of Washington Press, 12-44.

Mandelbaum J. (2013). Storytelling in Conversation. In J. Sidnell, T. Stivers (eds). The Handbook of Conversation Analysis, Oxford : Wiley-Blackwell, 492-507.

Marranconi F., Kerbage H. (2017). Les services de santé mentale pour les réfugiés syriens. Hommes et Migration 1319, 87-94.

Maitilasso, A. (2014). Raconte-moi ta migration. Cahiers d'études africaines, 213-214, 241-265.

Mekdjian S. (2016) Les récits migratoires sont-ils encore possibles dans le domaine des Refugee Studies ? Analyse critique et expérimentation de cartographies créatives. ACME: An International E-Journal for Critical Geographies, University of British Columbia, Okanagan, 15 (1).

Pekarek Doehler S., De Stefani E., Horlacher A. S. (2015). Time and Emergence in Grammar. Dislocation, topicalization and hanging topic in French talk-in-interaction. Amsterdam: John Benjamins.

Sacks H. (1974). On the Analysability of Stories by Children. In R. Turner (Ed): Ethnomethodology. Selected Readings. Harmondsworth: Penguin: 216-323.

Sacks H. (1978). Some technical considerations of a dirty joke. In J. Schenkein (Ed.), Studies in the Organization of Conversational Interaction, New York : Academic Press, 249-270.

Sacks H. (1992). Lectures on Conversation, Oxford, Blackwell.

Saglio-Yatzimirski M.-C. (2018). La voix de ceux qui crient. Paris : Albin Michel.

Stivers, T. (2008). Stance, alignment, and affiliation during storytelling : When nodding is a token of affiliation. Research on Language and Social Interaction, 41 (3), 31-57.

Traverso Véronique (1994). Les récits de la confidence, in Brès J. (éd), Le récit oral, Montpellier : Praxiling, $227-$ 237.

Traverso Véronique (1996). La conversation familière. Lyon : PUL. 
Traverso V. (2002), “De la variabilité des usages en interaction à des descriptions linguistiques réutilisables:

l'exemple de wa-llah", Cahiers de praxématique 38, p. 145-174.

Traverso V. (2003). Aspects de la négociation dans un polylogue. Etudes Romane, 54 Copenhague, 11-31.

Traverso V. (2005). Quelques formats intégrant la répétition comme ressource pour le développement thématique dans la conversation ordinaire. Rivista di Psicolinguistica Applicata (Special Issue on Conversation Analysis, R. Galatolo et A. Fasulo (dir)), 153-166.

Traverso V. (2014). Positionnements identitaires multiples dans une réunion plurilingue : traces dans l'interaction et effets structurants. In Greco L., Mondada L., Renaud P. (eds)), Identités en interaction, Limoges: Lambert-Lucas, 67-87.

Traverso V. (2017). Les frontières au prisme de la catégorisation. Construire et déconstruire de la différence : une discussion à propos de l'usage du hammam in Auzanneau M., Greco L. (éds), Dessiner les frontières, Lyon : ENS Editions.

Traverso V. (2019), Sessions de soutien psychosocial avec des femmes syriennes réfugiées au Liban, Rhizome, 73(3), p. 9-10.

Traverso V., Kerbage H. (2020). Life skills et awareness sessions (2) "Répondre à son tour" ; (3) "Faire celle qui sait" ; (4) "L'hygiène, principes et décalages". Carnet de I'Ifpo. https://ifpo.hypotheses.org/

Traverso V. (à paraître). Construire un sens aux récits dans des séances de suivi psychologique avec des femmes migrantes, in Chambon N. \& Traverso V. (dir), Raconter, relater, traduire : paroles de la migration, Lambert Lucas.

\section{Conventions de transcription}

Pour faciliter la lecture, la mise en forme de la transcription utilise :

- des caractère normaux (Courrier) pour la transcription

- des caractères plus fins (Courier) pour les traductions

- des caractère gras gris pour les notations multimodales.

Les conventions utilisées sont les conventions ICOR http://icar.cnrs.fr/projets/corinte/documents/2013_Conv_ICOR_250313.pdf

\begin{tabular}{|c|c|c|c|}
\hline$A B C$ & \multicolumn{3}{|c|}{$\begin{array}{l}\text { le pseudonyme du participant est indiqué par trois lettres en majuscules. Par souci de simplification, les } \\
\text { médecins apparaissent comme DOC et les interprètes comme IPA }\end{array}$} \\
\hline [ ] & début et fin du chevauchement & $x x x$ & segment inaudible \\
\hline par- & troncation & ハ & intonation montante/ descendantel \\
\hline : & allongement &.$h$ & aspiration \\
\hline (.) & pauses non chronométrées $(<0.2 \mathrm{~s})$ & (il va) & transcription incertaine \\
\hline (2.2) & pauses chronométrées (en secondes) & ${ }^{\circ}$ bon ${ }^{\circ}$ & voix basse ou très basse $\left({ }^{\circ}{ }^{\circ}\right.$ bon $\left.^{\circ \circ}\right)$ \\
\hline$\&$ & continuation du tour de parole & ALORS & volume augmenté ou autre marque d'insistance \\
\hline & enchaînement rapide & {$[\ldots]$} & coupure due au transcripteur \\
\hline ((rire)) & phénomènes non transcrits & $->$ & $\begin{array}{l}\text { avant le pseudonyme, indique les tours } \\
\text { commentés dans l'analyse }\end{array}$ \\
\hline
\end{tabular}

La transcription de l'arabe est faite suivant la convention ARAPI

https://ifpo.hypotheses.org/933

Dans la traduction, le signe \{\} entoure des adaptations visant à faciliter la compréhension (des ajouts ou la traduction par des équivalents pragmatiques par exemple pour les marqueurs discursifs).

Conventions pour la notation des gestes s'inspirent librement de celles développées par $\mathrm{L}$. Mondada, consultables sur le site CORVIS http://icar.univ-lyon2.fr/projets/corvis/.

\begin{tabular}{|l|l|}
\hline abc & $\begin{array}{l}\text { L'utilisation de minuscules dans la colonne des pseudonymes indique que la ligne est consacrée à une } \\
\text { description de gestes ou de mouvements }\end{array}$ \\
\hline tourne la tête & Les lignes de description des gestes sont en caractère gris \\
\hline bis & Indque que le geste précédemment décrit est répété \\
\hline & \multicolumn{2}{|c|}{$\begin{array}{l}\text { Dans la transcription indique le début et la fin du geste ou du regard décrit à la ligne suivante. La } \\
\text { notation des gestes des médecins utilise toujours le symbole * }\end{array}$} \\
\hline$* *$ &
\end{tabular}




\begin{tabular}{|l|l|}
\hline$(())$ & $\begin{array}{l}\text { Dans certains extraits, les descriptions de gestes sont insérées dans la transcription entre doubles } \\
\text { parenthèses }\end{array}$ \\
\hline >> ou --> & Indique que le geste continue aux lignes suivantes jusqu'à la borne suivante \\
\hline
\end{tabular}

